\title{
Impact of stochastic representations of pedestrian actions on serviceability response
}

\author{
C. Ramos-Moreno ${ }^{1}$, A.M. Ruiz-Teran ${ }^{2}$, and P.J. Stafford ${ }^{3}$ \\ ${ }^{1}$ Senior Bridge Engineer, Arcadis Consulting, UK \\ ${ }^{2}$ Senior Lecturer, Dept of Civil \& Environmental Engineering, Imperial College, London, UK \\ ${ }^{3}$ Reader, Dept of Civil \& Environmental Engineering, Imperial College, London, UK
}

\begin{abstract}
Over the past 15 years, there have been some research outcomes in other disciplines, that could be used to produce new, more accurate, and realistic numerical models to characterise pedestrian loads and to significantly improve predictions of response for multiple pedestrian scenarios. However, the disconnection between fields has not facilitated this further research. Using this, the paper presents (1) a new sophisticated load model that includes the description of vertical and lateral loads, including pedestrian-structure interaction, (2) the numerical description of relationships to describe the key parameters of the proposed model, and (3) the evaluation of the effects of pedestrian characteristics that are relevant for serviceability response of footbridges. The proposed new load model enables inherent variability of individual pedestrian actions (intrasubject variability), probabilistic description of how pedestrian characteristics vary among subjects (inter-subject variability), and collective human behaviour (pedestrian-pedestrian interaction). Some of these characteristics are not currently considered in design approaches and can have a substantial impact upon structural response assessment. Finally recommendations are made for many of these characteristics to be introduced in analyses to evaluate the vibration serviceability limit state of footbridges in a more accurate and realistic manner.
\end{abstract}

\section{Introduction}

Footbridges are becoming lighter and slender structures, with designs governed by the serviceability limitstate for vertical (e.g., Bachmann 1992, Bachmann and Ammann 1987) and lateral vibrations (e.g., Fujino et al. 1993, Dallard et al. 2001). A satisfactory prediction of this response relies upon describing human actions accurately and realistically. The responses of these bridges are analysed following deterministic approaches and several works have argued that some of these approaches may be inconsistent with reality, e.g., Zivanović et al. (2007). The parameters that are commonly used to define anthropogenic loads are characterised by a significant degree of inherent variability: the wide range of anthropometric characteristics that exist within the human population (inter-subject variability), the inability of individual subjects to repeat monotonous activities with constant features such as step length or frequency (intra-subject variability) as described in Giakas and Baltzopoulos (1977), and the different constraints that arise from collective behaviour (interactions between subjects).

This article presents a novel pedestrian load model that is capable of representing the aforementioned components of variability in a realistic manner. It also examines the impact of considering these aspects in a rigorous manner as opposed to ignoring them or representing them in an overly-simplified way. Its original contributions are: (1) an overview of the limitations and drawbacks of existing models, and the recently published research in other fields that could be used to address some of these problems; (2) a description of the new model for vertical and lateral loads, highlighting its advantages compared to current models; (3) a description of the intra-subject variability of human vertical and lateral loads; (4) an investigation of the inter-subject variability of pedestrian loads; (5) a proposal for the evaluation

Cite as: Ramos-Moreno, C., A.M. Ruiz-Teran \& P.J. Stafford (2020). Impact of stochastic representations of pedestrian actions on serviceability response. Proceedings of the Institution of Civil Engineers - Bridge Engineering,

https://doi.org/10.1680/jbren.19.00050 
of multiple-pedestrian scenarios including collective behaviour; and (6) the implementation of all these characteristics in a single pedestrian load model. The model is sophisticated due to the number of variables that it intends to represent. Therefore, the authors propose to use this model for structures where their importance (e.g., location, importance, number of users, etc.) substantiates the need of accuracy for the assessment of the serviceability response prior to the construction of the structure. The model lends itself to be implemented by developers of structural design software packages as background routines, in which case the model could be used in any structure, regardless of their importance.

\section{Current load models: inherent drawbacks and recent advances}

Current codes and guidelines evaluate the vibration serviceability limit state through simplified methods that facilitate their implementation in practice. However, comparisons between the response obtained via these prescribed methods and empirical data have a poor agreement. The non-trivial discrepancies can be explained by the simplicity of the load models (often resembling the very first proposals in this field) and the fact that the prescriptions do not reflect the significant body of relevant research that has been published in recent years.

Generally speaking, the prescriptions of current codes and guidelines (e.g., Synpex 2008 or SETRA 2006) are mainly based upon deterministic approaches that reflect vertical and lateral actions applied by a single pedestrian. These vertical and lateral loads are described mathematically through a Fourier series (Eq. 1) that represents the total load transmitted by both feet in time $p(t)$.

$$
p(t)=W_{p}\left[1+\sum_{n=1}^{\infty} b_{n} \sin \left(\omega_{n} t-\phi_{n}\right)\right]
$$

where $b_{n}$ is the amplitude of each harmonic with frequency $\omega_{n}$ and phase $\phi_{n}$, and $W_{p}$ the static weight of the pedestrian. This representation is very similar to that proposed by Blanchard et al. in 1977 for vertical loads, which was adopted in design codes (BSI, 1978). Their simplicity was partly driven by the computational capabilities of the time. However, revised versions continued to be used in the $21^{s t}$ century (BSI, 2006). As a result, a number of researchers focused attention on the estimation of the harmonic parameters $\left(b_{n}, \omega_{n}\right.$ and $\left.\phi_{n}\right),(e . g$. . Bachmann et al. 1995, Rainer and Pernica 1986, Kerr 1998, Butz et al. 2008).

Harmonics describing lateral loads initially got very little attention (despite the warnings given by Bachmann and Ammann 1987, Fujino et al. 1993), due to their smaller magnitudes. Further research (Ingólfsson et al. 2012) was undertaken following the excessive lateral response observed in the Millennium footbridge in London (Dallard et al. 2001), among others. Later proposals based upon the representation of human movement using inverted pendulums have gained popularity (e.g., Macdonald 2009, Carroll et al. 2013). These models derive lateral load amplitudes from the movement of the pedestrian Centre of Mass $(\mathrm{CoM})$ and allow pedestrian structure interaction to be accommodated.

The appraisal of the vertical and lateral effects caused by scenarios with multiple pedestrians have also evolved considerably. Blanchard et al. (1977) initially suggested that a single pedestrian inducing a resonant response could be used for representing the critical scenario for serviceability (approach that circumvented the need to consider simulations for crowd movement). This assumption was subsequently adopted in codes (e.g., BSI, 1978) and other researchers (e.g., Ellingwood and Tallin 1984). Later, several researchers suggested approaches using multiple pedestrians (e.g., Matsumoto et al. 1978).

Fourier amplitude spectra of the loads induced by individual pedestrians show that the applied loading has a very strong contribution at a frequency corresponding to the mean step frequency and its harmonics, but that significant contributions are also distributed around these. Researchers have attempted to implement this intra-subject variability into the simplified framework shown in Eq. 1 by; (1) including additional terms in the definition of pedestrian loads for vertical (Brownjohn et al. 2004) and lateral (Ingólfsson et al. 2011) components; (2) adjusting the principal Fourier amplitudes to account 
for the impulse distributed among proximal frequencies, for vertical (Ebrahimpour and Sack 1992) and lateral (Ricciardelli and Pizzimenti 2007) loads; and (3) using random phase angles, $\phi_{n}$, for the multiple harmonics to represent the variability of consecutive steps (Ingólfsson and Georgakis 2011).

The impact of considering differences among pedestrians (inter-variability) was also explored by Matsumoto et al. (1978) and others discussed in Pedersen and Frier (2010). More recently, a guideline has considered this inter-variability in the definition of the harmonics amplitude to define the vertical loads (Millford and Young 2006) and other research groups have explored its definition through statistical analysis (e.g., Chen et al. 2019). Evaluations found in SETRA (2006) or BSI (2008) illustrate how descriptions similar to these earlier studies are still adopted. Others have suggested reproducing the stochastic nature of pedestrians by using normal distributions for step lengths, and pedestrian weights (e.g., Pedersen and Frier 2010).

The simulation of traffic flows including collective behaviour has been developed more recently. For vertical response, harmonic amplitudes have been defined as a function of the size of the group of pedestrians (Ebrahimpour and Sack 1992), described with parameters that vary to reflect the flow density (Brownjohn et al. 2004), and Monte Carlo simulations have been used. This approach appears in the guidelines of Synpex (2008) and Setra (2006). For lateral response, the proposals aim to recreate the empirically observed phenomenon of synchronisation between pedestrians and the lateral vibration of the bridge. Fujino et al. (1993) pointed out that loads expressed as in Eq. 1 were inadequate for the prediction of lateral structural movement as the amplitudes of lateral loads increase with increasing lateral movement of the bridge. Various researchers proposed methods to capture this effect (e.g., Venuti et al., 2007, or, more recently, Mulas et al. 2018).

Despite the progress described above, the main shortcomings with the current approaches are: (1) truncated Fourier series do not allow explicit consideration of the impulse introduced by individual footsteps, (2) Fourier series are unable to capture the phenomenon of pedestrian-structure synchronisation, (3) Fourier-based approaches are not well suited to represent intra-subject variability, (4) the assumption of deterministic parameters (e.g. $W_{p}$ or $b_{n}$ in Eq. 1) to describe all the population is clearly a gross simplification that ignores the variances and covariances among these parameters, and (5) basic Monte Carlo simulations need refinement for high-density flows to account for important pedestrian interactions. In an attempt to shed light on this state-of-affairs, this study introduces a model capable of addressing these issues, as it is based upon the definition of the impulse transmitted by individual footsteps, is able to include the variability of consecutive steps in a meaningful manner, defines pedestrian loads according to different characteristics such as speed, step frequency, pedestrian mass, etc., and reproduces the effects of interactions among different pedestrians (such as overtaking, change of direction, groups, etc.).

\section{Definition of the new load model}

The primary purpose of this study is to propose a realistic model for vertical and lateral pedestrian loads capable of reflecting aspects such as intra- and inter-subject variability as well as crowd flow interactions. In order to model these effects in a realistic manner, the proposed load model defines the temporal evolution of vertical and lateral load amplitudes associated with each footstep as functions of the gait and physical characteristics of individual pedestrians. This description of individual loads in time can then be used to describe the loads transmitted by multiple pedestrians who may interact to avoid one another by changing their speed, direction of movement, etc.

\subsection{Vertical Load Model}

Several studies from the area of physical medicine define relationships between the vertical displacement of the CoM of a human body and the step length adopted by that person e.g., Gard and Childress (2001), as well as between the walking speed and the anthropometric characteristics of the pedestrian e.g., Nilsson and Thorstensson (1987). These studies support the intuition that individual pedestrians adopt 


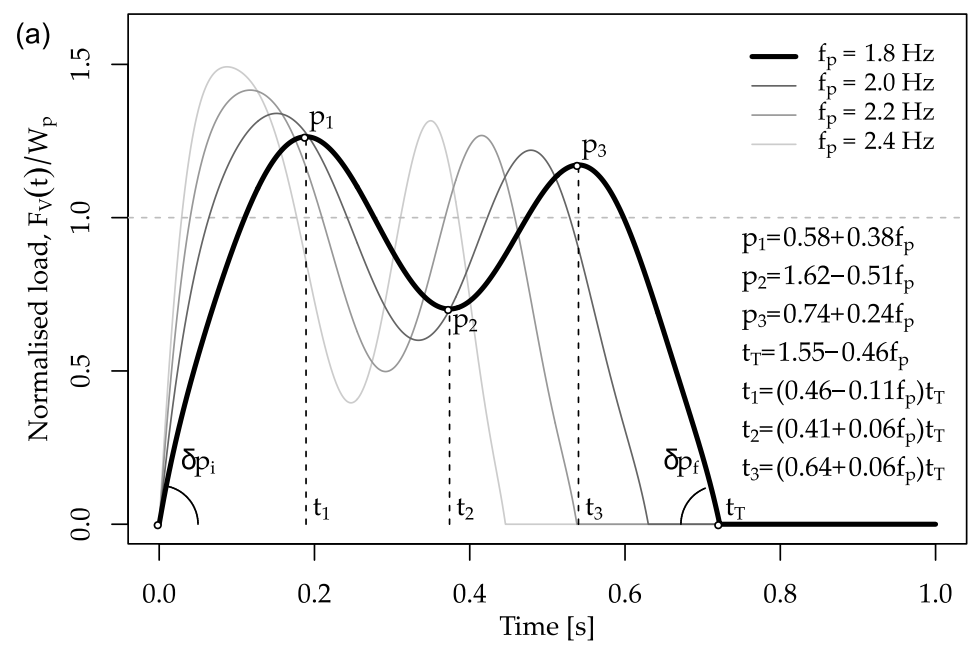

(b)

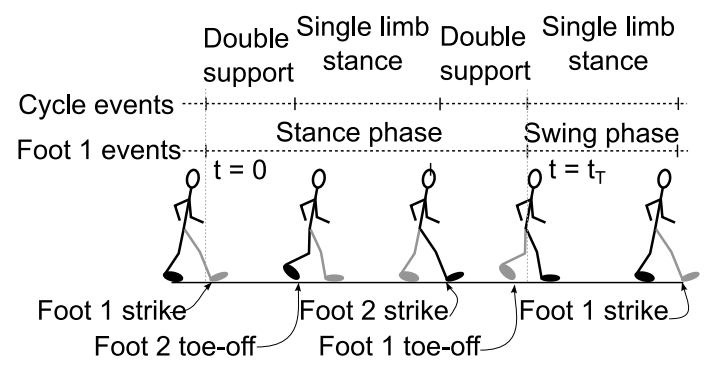

Figure 1: (a) Time-variation of the vertical load amplitude for a single footstep. The initial and final slopes are defined as $\delta p_{i}=400.16-670.98 f_{p}+362.55 f_{p}^{2}-60.41 f_{p}^{3}$ and $\delta p_{f}=4.54-9.15 f_{p}$, respectively. Empirical data from Butz et al. (2008). (b) Correlation between the position of the foot during the walking cycle, as described by Rose and Gamble (1994), and vertical loads described in (a).

a combination of speed and step length or step frequency depending upon their physical characteristics, consequently defining the vertical load transmitted by each foot (Rose and Gamble 1994).

\subsubsection{Vertical load amplitudes $\left(F_{v}\right)$}

A comprehensive empirical dataset, including load-time profiles of a large number of experimentally recorded footsteps, was presented by Butz et al. (2008) and used to define the relationship between gait characteristics and vertical load amplitudes. The recorded ground reaction force is first normalised by the weight of the subject $\left(W_{p}\right)$ and its temporal variation (see Fig. 1a) can be expressed using nine parameters: peaks $p_{1}$ and $p_{3}$, local minimum $p_{2}$, initial and final slopes $\delta p_{i}$ and $\delta p_{f}$, and the times $t_{1}, t_{2}, t_{3}$ and the total time $t_{T}$. These parameters describe a vertical load shape that is related to the walking cycle of the subject and to the movement described by his centre of mass, as shown in Fig. 1b.

Butz et al. (2008) shows that all the parameters in Fig. 1a have a clear dependence upon the step frequency $f_{p}$. Loads generated while walking with low step frequencies $(\approx 1.6 \mathrm{~Hz})$ have relatively broad and shallow peaks and, to first-order, resemble rectangular pulses, whereas loads generated with higher step frequencies $(>2 \mathrm{~Hz}$ ) have more pronounced narrow peaks. These trends are experimentally corroborated by the observations of many previous researchers (e.g., Keller et al. 1996). Median estimates of the 9 parameters are defined in Fig. 1a. The standard deviations of the distributions that describe these nine parameters are $\sigma_{t_{T}}=0.05[\mathrm{~s}], \sigma_{t_{1}}=0.05[-], \sigma_{t_{2}}=0.03[-], \sigma_{t_{3}}=0.05[-], \sigma_{p_{1}}=0.16[-]$, $\sigma_{p_{2}}=0.12[-], \sigma_{p_{3}}=0.14[-], \sigma_{\delta p_{i}}=4.9[-], \sigma_{\delta p_{f}}=2.2[-]$.

The ratio of the vertical load amplitude for a single footstep to the pedestrian weight can be defined as a $8^{t h}$ order polynomial, $F_{v}(t) / W_{p}=\sum_{i=0}^{8} a_{i} x^{i}$, which depends on the step frequency. However, a $8^{\text {th }}$ order polynomial does not facilitate analytical solutions. For that reason, the ratio $F_{v}(t) / W_{p}$ has been represented in this work through the use of three half-sine waves: the first models the heel strike (capturing $p_{1}$ and $t_{1}$ ), the third reflects the foot pushing off the ground (defined by $p_{3}, t_{3}$ and $t_{T}$ ), and the second represents the transition between these phases (representing $p_{2}$ and $t_{2}$ ). This simplification is very accurate (differences in peak accelerations are below 5\%), computationally faster, and allows for analytical solutions to be defined. 


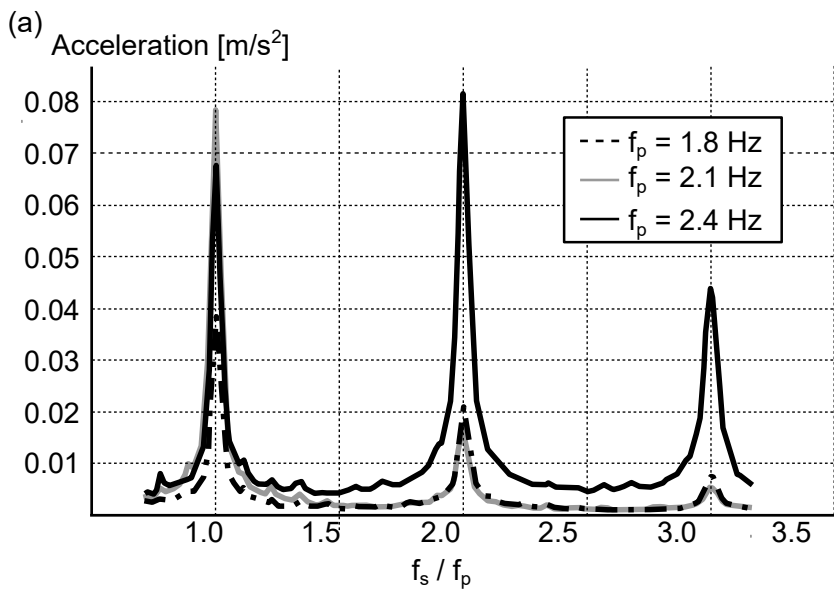

(b) Acceleration $\left[\mathrm{m} / \mathrm{s}^{2}\right]$

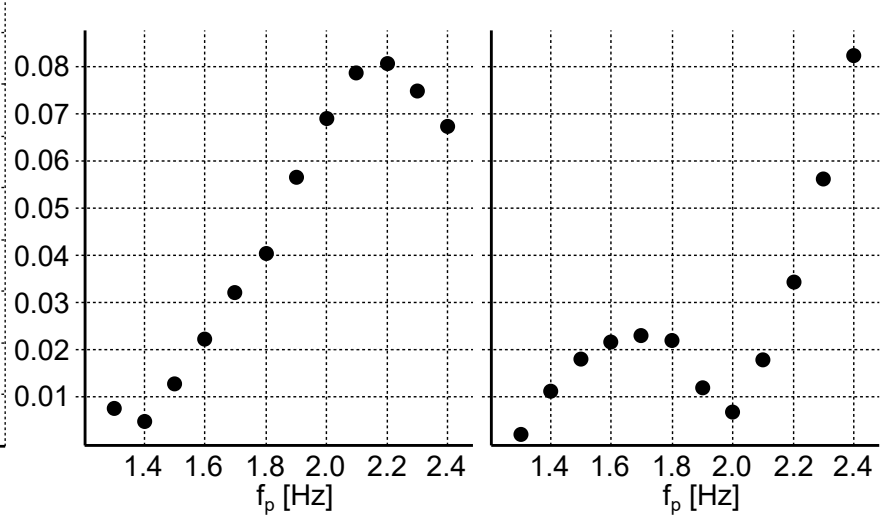

Figure 2: Vertical maximum acceleration generated by loads defined with the proposed model: (a) versus $f_{s} / f_{p}=1$ for $f_{p}=1.8,2.1$ and $2.4 H z$; (b) versus $f_{p}$ for $f_{s} / f_{p}=1$; and (c) versus $f_{p}$ for $f_{s} / f_{p}=2$.

\subsubsection{Response predicted for different step frequencies}

In order to assess the effects of the proposed load model, we compare numerical acceleration responses for a set of simply supported bridges under the action of a single pedestrian walking at a constant step frequency $f_{p}$ for each analysis. With the aim of analysing non dimensional parameters, there is a constant bridge-to-pedestrian weight ratio and the pedestrian makes the same number of steps when crossing the bridge (achieved by adjusting the velocity of the pedestrian). Fig. 2a shows a comparison of the accelerations obtained at midspan plotted against the ratio of the structural frequency, $f_{s}$, and the pedestrian step frequency, $f_{p}$. This resonance parameter $\left(f_{s} / f_{p}\right)$ helps to identify cases when severe acceleration demands are expected. When $f_{s} / f_{p}=1$, the pedestrian load excites the fundamental bridge frequency and reinforces the response at every cycle of vibration. When it is equal to 2,3 , etc. the response is reinforced every $2^{\text {nd }}, 3^{\text {rd }}$, etc. cycle. Figs. 2(a-c) demonstrate the marked differences in response that can be expected due to the temporal variation of load depending upon the step frequency. When walking at a fast pace, the accelerations generated by pedestrian loads increase in magnitude (as shown in Figs. 2(b-c)) as the peaks $p_{1}$ and $p_{3}$ described in Fig. 1a are larger and occur closer in time to one another.

\subsubsection{Comparison with existing models}

The responses obtained with the proposed load model and from using the Fourier-based models contained in the SETRA guideline (2006) (similar to the UK prescriptions, BSI, 2008) and the Synpex guideline (2008), both representing one pedestrian, are now compared for a broad range of simply-supported bridges adopting the same assumptions presented in the previous section. The load model within the SETRA guideline is independent of the pedestrian step frequency, while the load model proposed in the Synpex guideline uses a truncated Fourier series with $f_{p}$-dependent amplitudes. The results from application of the SETRA method (Fig. 3a) show a single peak with amplitudes similar to the Synpex model when $f_{p}=f_{s}$, but do not exhibit resonant peaks otherwise. The Synpex model generates results that appear to be only weakly dependent upon the step frequency for most cases, but generally shows a qualitative agreement with the results of Fig. 2a.

To quantify differences in the accelerations that arise from application of an existing model and that of the present study, a more realistic analysis scenario involving the passage of crowds of pedestrians 
(a)

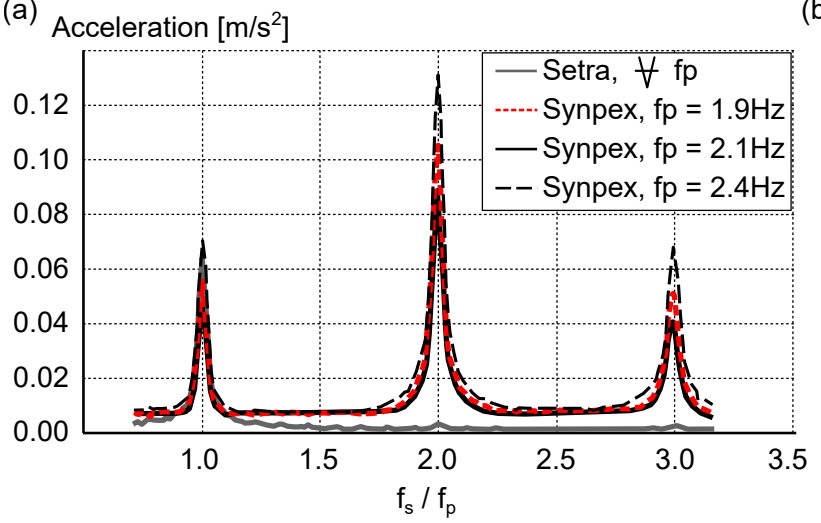

(b)

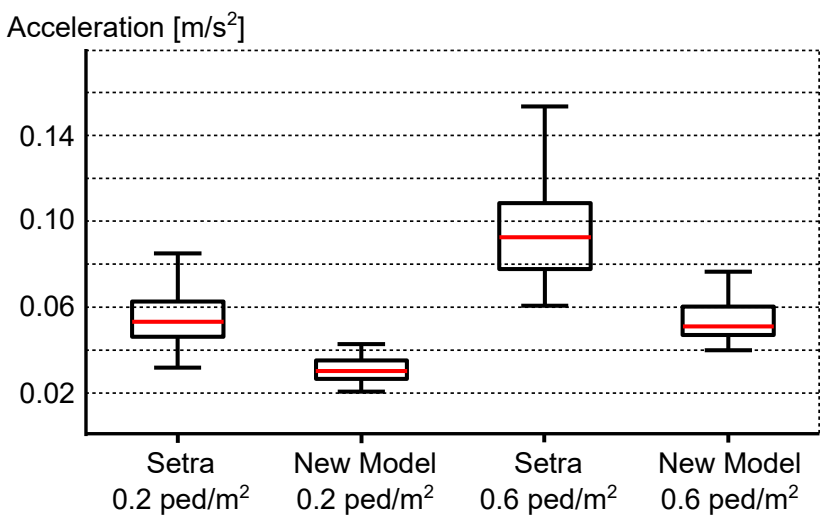

Figure 3: Maximum vertical accelerations generated with current load models (SETRA and Synpex guidelines) for: (a) an individual pedestrian, (b) crowd flows (50 simulations per case) with densities of $0.2 \& 0.6 \mathrm{ped} / \mathrm{m}^{2}$.

(with pedestrian densities of 0.2 and $0.6 \mathrm{ped} / \mathrm{m}^{2}$ ) was considered. Fig. 3b presents the results (using box and whisker plots that show medians, the inter-quartile range and the range containing $99 \%$ of the results) obtained from the SETRA model and our model for multi-pedestrian crowd flows crossing a simply-supported bridge with a fundamental frequency fs $=2.0 \mathrm{~Hz}$. For each model 50 analyses were run in which individual pedestrians are allocated a constant step frequency, that they maintain while crossing the bridge, obtained as a random variate from a normal distribution of step frequencies defined as $f_{p} \sim N\left(f_{s}, 0.175 \mathrm{~Hz}\right)$. Pedestrian arrival times are described by a Poisson process. It should be noted that for the single-pedestrian case the SETRA model produces almost identical results to our model when $f_{s} / f_{p}=1$ and $f_{p}=2.0 \mathrm{~Hz}$ (see Figs. $2 \mathrm{a}$ and $3 \mathrm{a}$ ). In addition, for the simulations both models use the same normal distribution to generate samples of $f_{p}$. Therefore, differences in Fig. 3b can only arise from the effects caused by pedestrians with $f_{p} \neq f_{s}$ (effectively ignored in the SETRA model - see Fig. 3a).

In summary, the proposed load model for vertical loads has several advantages over methods within codes and guidelines. It is able to reproduce the effects caused by loads of different step frequencies and it captures the effects of these loads under non-resonant conditions (as opposed to Fourier series models with one term defined in the SETRA guideline). Both the new model and that proposed in the Synpex guideline predict similar results for loads at similar step frequencies (the basis of the new model is the same as that of this guideline), however the new model is more easily able to reproduce intra-subject variability, due to the use of a Fourier series representation in Synpex.

\subsection{Lateral Load Model}

Lateral loads induced by pedestrians have historically been described using Fourier series (e.g. Butz et al. 2008, Charles et al. 2006, Ricciardelli and Pizzimenti 2007, Ingólfsson and Georgakis 2011). However, as opposed to studies of vertical movement, the prediction of lateral loads cannot simply be attributed to characteristics of the gait such as step frequency (Butz et al. 2008). Amplitudes of pedestrian induced lateral loads have been recorded experimentally by several researchers (e.g., Butz et al. 2008, Nilsson and Thorstensson 1989), concluding that they are characterised by significant variability, but no quantitative relationship between these magnitudes and gait characteristics (speed, step frequency, or width of the step adopted) are provided.

Several authors indicate an association between the lateral displacement of the centre of mass (CoM) and the position of the foot (lateral step width). Mackinnon and Winter (1993) experimentally observed 


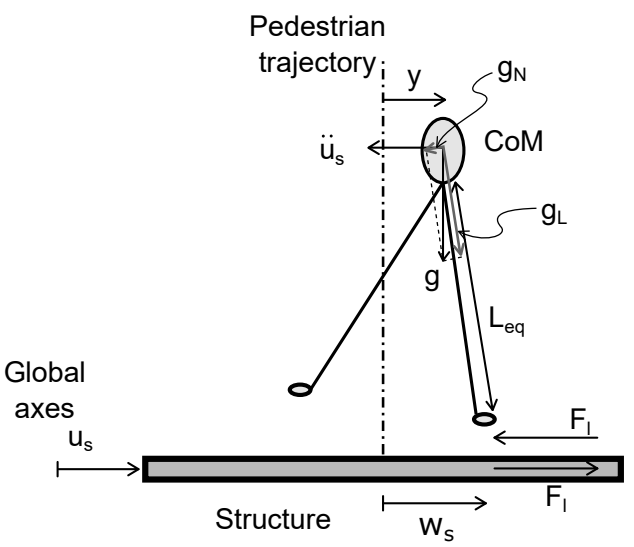

Figure 4: Lateral equilibrium of a pedestrian represented as an inverted pendulum $\left(u_{s}\right.$ is the lateral bridge deck displacement).

that equilibrium of the human body in the lateral direction was ensured by the correct positioning of the foot in relation to the CoM, as it had been initially stated by Townsend (1985) on the basis of a theoretical study using an inverted pendulum model. Alternative approaches have also been introduced recently (e.g. Macdonald 2009). The works of Townsend (1985) and Macdonald (2009) (empirically corroborated by Carroll et al. 2013) formulate a theoretical basis for describing how movements of the CoM relate to modification to gait characteristics as well as the positioning of steps among other parameters.

\subsubsection{Lateral load magnitudes $\left(F_{l}\right)$}

Townsend (1985) and Macdonald (2009) both describe the lateral position of a pedestrian's CoM in time by representing the subject as an inverted pendulum (see Fig. 4). The model has been used extensively in the field of biomechanics, but its application in structural engineering has only occurred recently. It is based upon the equilibrium of forces in the frontal plane. Given the acceleration of the bridge $\left(\ddot{u}_{s}\right)$, the maximum height of the CoM ( $L_{e q}$, which is the length of the inverted pendulum equal to 0.6 times the pedestrian height, Eames et al. 1999), the pedestrian mass $\left(m_{p}\right)$, and the position $(y)$ and acceleration $(\ddot{y})$ of the CoM in local coordinates, Eqs. 2-3 allows one to define next foot position (described by $w_{s}$, which is half of the step width), and the resulting lateral load $\left(F_{l}\right)$. The second derivative of the transverse pedestrian trajectory over the time should be considered in the sums if it is not negligible.

$$
\begin{gathered}
\ddot{u}_{s}+\ddot{y}=-g_{N}=-\frac{g}{L_{e q}}\left(w_{s}-y\right) \\
F_{l}=-m_{p}\left(\ddot{u}_{s}+\ddot{y}\right)=m_{p} \frac{g}{L_{e q}}\left(w_{s}-y\right)=m_{p} \Omega_{p}^{2}\left(w_{s}-y\right)
\end{gathered}
$$

Lateral loads defined with this model have one minor shortcoming in that they cannot reproduce empirically observed loading conditions when both feet of a subject are in contact with the ground, the double stance phase, (Nilsson and Thorstensson 1989). Nevertheless, this deficiency has very little effect upon the results given that accelerations of the CoM are small during this phase. The crucial feature of this lateral load model is the ability to include feedback between the accelerations of the platform and the lateral load amplitudes. Loads defined in this manner are able to generate large contributions to the overall acceleration response at both the step and structural frequencies, as has been experimentally observed (e.g., Carroll et al. 2013, Ingólfsson et al. 2011). 
(a)

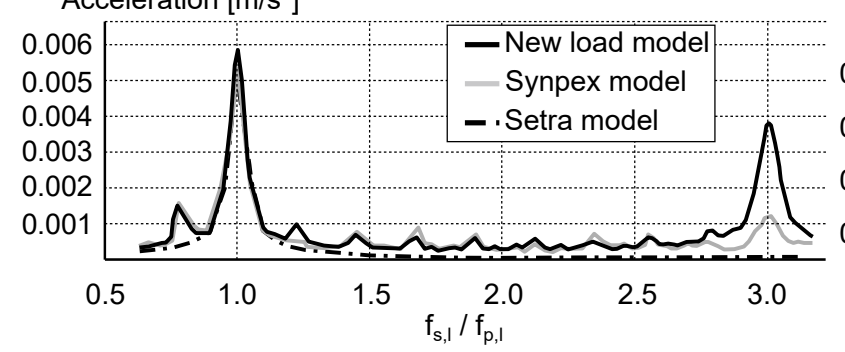

(b)

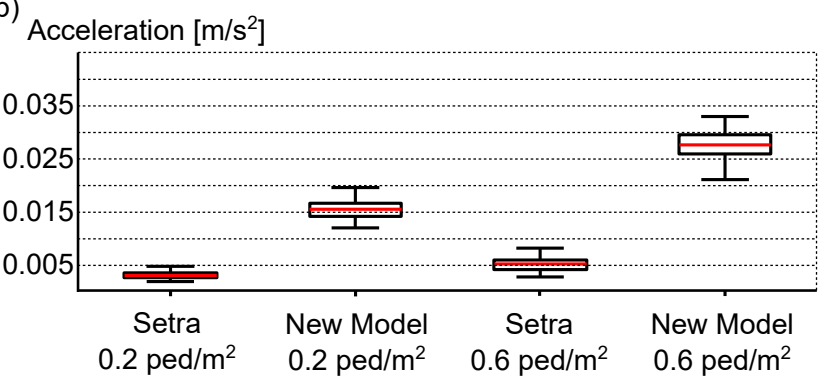

Figure 5: Maximum lateral mid-span accelerations of simply supported bridges for: (a) an individual pedestrian (results valid for all $f_{p, l}$ ), (b) crowd flows with densities of $0.2 \& 0.6 \mathrm{ped} / \mathrm{m}^{2}$.

\subsubsection{Response predicted for different step frequencies and comparison with guidelines}

Fig. 5a shows the maximum accelerations induced in simply-supported bridges with different lateral frequencies, $f_{s, l}$, based on our new model. The loads correspond to a $1.70 \mathrm{~m}$ tall subject walking with a fixed step frequency, $f_{p, l}\left(f_{s, l} / f_{p, l}=2\right)$, that uses the same step width $(0.103 \mathrm{~m})$ regardless of the step frequency and speed adopted while walking. This step width is representative of multiple experimental measurements and is related to the subject height. A range of structures (similar to those described in Section 3.1.2) and pedestrians are considered, and the ratio of pedestrian-to-bridge weight and the number of steps taken remain constant. The results of our model presented in Fig. 5a now show resonant response for $f_{s, l} / f_{p, l}=1$ and $f_{s, l} / f_{p, l}=3$ (and any other odd integer), which is due to the alternating signs of the loads associated with consecutive steps. As opposed to the vertical loads where we had resonance for even $f_{s, l} / f_{p, l}$ ratios, these combinations now cause interference. The results presented in Fig. 5a allow an important conclusion to be drawn: for a given ratio of structural to pedestrian frequencies, $f_{s, l} / f_{p, l}$, and a particular step width and pedestrian height, the acceleration response is independent of the step frequency adopted. This can be explained by the fact that the impulse introduced by the lateral loads is the same, regardless of the lateral frequency $f_{p, l}$. In the vertical direction this statement is not valid due to the different shapes of the vertical loads according to $f_{p}$.

Fig. 5a also compares the results of the new model with those obtained using the SETRA and Synpex guidelines. The three of them provide very similar results for $f_{s, l} / f_{p, l}=1$. However, SETRA model provide negligible lateral accelerations for essentially all other ratios, as a result of the Fourier approach adopted by the guideline. The Synpex guideline show very similar results to our model for non-resonant ratios. However, the peak at $f_{s, l} / f_{p, l}=3$ has a much lower amplitude, as a result of Synpex considering lateral loads with a rectangular shape (with an amplitude of $0.04 W_{p}$ ) and without consideration of any structural feedback. The observed differences in the results obtained when considering single pedestrians crossing the bridge are exacerbated for multi-pedestrian scenarios (see Fig. 5b). Pedestrian arrivals follow a Poisson process, and subjects walk with a constant vertical step frequency drawn from a normal distribution $\mathrm{N}(2,0 ; 0,175 \mathrm{~Hz})$ on a structure with $f_{s, l}=1.0 \mathrm{~Hz}$, with the step width and height defined previously when discussing Fig. 5a. Median accelerations produced with loads defined by the proposed model are more than 5 times larger than those predicted from the model proposed by the SETRA guideline. Therefore loads of non-resonant pedestrians $\left(f_{s, l} / f_{p, l} \neq 1\right)$ produce dramatic differences in the structural response.

\section{Parameters of the proposed load model}

To obtain a robust estimate of the structural response, the conceptual framework for the load model must be sound and the actual parameters of the model must also be rigorously defined. The purpose of the 

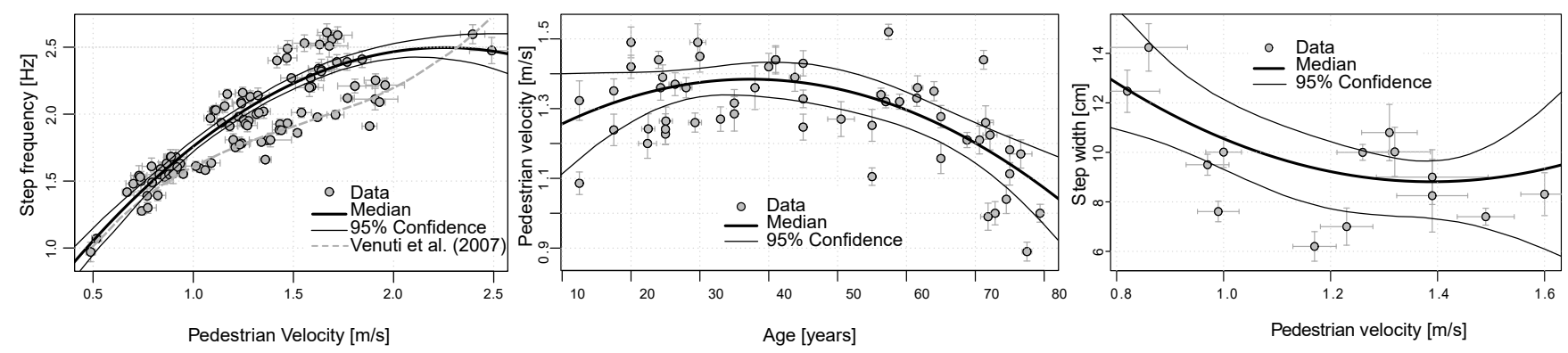

Figure 6: Datasets from the meta-analyses: a) Step frequency $f_{p}$ against pedestrian velocity $v$, b) Free pedestrian velocity against age (model predictions use expected heights for each age), and c) Pedestrian step width $w_{s, t}$ against velocity.

present section is to detail how the relevant parameters of the new load model have been calibrated.

The dataset used to calibrate the model parameters combines data from a number of experimental programs, mainly focused on physical medicine and rehabilitation. In general, each source study reports data that reflect groups or subgroups of individual subjects rather than data for each examined individual. Hence, graphical representation of this data will provide one point for each subgroup with error bars that correspond to the mean and standard error for that particular subgroup. Data used to describe the definition of step frequency is originally from studies that include pedestrians from Japan, USA and Western Europe. Almost all these studies together with research using pedestrians from Canada and Central Europe describe the database for the velocity. Finally, the relationship for the step width uses several experimental works in common with the previous relationships as well as 13 others that describe results for populations of countries located in Western Europe or the USA. While the datasets used to constrain each parameter differ, we assume that the underlying anthropometric characteristics are equivalent in each case. In total, data from 28 different studies was compiled. The specific details of the studies can be found in Ramos-Moreno (2016).

\subsection{Step frequency}

The parameter that most readily characterises walking for different traffic conditions is the pedestrian speed, yet for the new load model the key parameter is the step frequency. Therefore, it is important to ensure that a robust relationship relates the step frequency that is adopted by a pedestrian to the typical speed of walking for a variety of traffic scenarios. Several researchers have attempted to quantify the relationship between step frequency and speed (e.g., Bertram and Ruina 2001). For the present study, we undertake a meta-analysis of a number of experimental studies looking at the correlation between step frequency and speed. Collectively, these studies provide information related to 909 different subjects. This dataset is represented in Fig. 6a in 33 bins. Vertical error bars describe the standard error of the mean step frequency and horizontal error bars reflect the standard error of pedestrian velocity in each bin.

The model for the expected step frequency, $f_{p}$, is described by a quadratic function of the speed, $v$ (Eq. 4, with $f_{p}$ in $\mathrm{Hz}$ and $v$ in $\mathrm{m} / \mathrm{s}$ ). The model was fitted using a weighted regression analysis in which the weights are inversely proportional to the variances of the binned data. The residual standard error of this model is 0.178 and together with the expected value of $f_{p}$ defines a normal distribution of step frequencies for a given pedestrian speed. Fig. 6a shows the proposed function of $f_{p}$ as a quadratic function of the speed and an alternative relationship derived by other researchers (Venuti et al., 2007)

Cite as: Ramos-Moreno, C., A.M. Ruiz-Teran \& P.J. Stafford (2020). Impact of stochastic representations of pedestrian actions on serviceability response. Proceedings of the Institution of Civil Engineers - Bridge Engineering,

https://doi.org/10.1680/jbren.19.00050 
for comparison. Both proposals have a general agreement, although the relationship proposed in this paper has been derived from a larger dataset and fits better all the observations. Thus, the relationship described in this paper is used to develop the pedestrian load model.

$$
f_{p}=0.11+2.11 v-0.47 v^{2}
$$

\subsection{Pedestrian velocity}

The model for the pedestrian velocity is based upon the research of Weidmann (1993) who suggested that the velocity naturally adopted by pedestrians, $v_{f}$, depends upon anthropometric characteristics (such as height) and age, and is then modified by external constraints such the aim of the journey and the density of the pedestrian flow, among others. Herein we adopt a similar procedure in which a pedestrian's velocity, $\mathrm{v}$, is defined as a function of their free velocity (in unrestricted conditions), $v_{f}$, and two factors representing the effect of the aim of the journey, $\phi_{j}$, and the flow density, $\phi_{d}$ (Eq. 5).

$$
v=v_{f} \phi_{j} \phi_{d}
$$

To obtain a model for the free velocity, we adopt a meta-analysis approach. The compiled dataset contains attributes from 1492 different pedestrians from 9 different countries. In Fig. 6b the developed model is shown along with the binned data recovered from the source studies. The model for free velocity is quadratic with respect to age $a_{p}$, and linear with height hp, as shown in Eq. 6. The standard deviation of this model is 0.087 .

$$
v_{f}=0.22+0.0128 a_{p}-\left(1.71 \times 10^{-4}\right) a_{p}^{2}+0.55 h_{p}
$$

Distributions of heights and ages of pedestrians expected to comprise pedestrian streams are routinely available for any given country.

Multiple researchers have evaluated the effect of the flow density on the mean speed adopted by pedestrians within the pedestrian flow. Data and trends suggested by Weidmann (1993) to reflect the effect of density on the mean speed are captured through the use of Eq. 7.

$$
\phi_{d}=1-\exp \left[-1.913\left(\frac{1}{d}-\frac{1}{5.4}\right)\right]
$$

where $\mathrm{d}$ describes the flow density $\left(\mathrm{ped} / \mathrm{m}^{2}\right)$. The data available from Ped-net.org (accessed 2013) (extracted from observation of pedestrians at different locations such as transport hubs or on the street) also allow one to infer how observed speeds are influenced by the purpose of the journey. Weidmann (1993) had previously recognised this effect and proposed the following values for the modifier $\phi_{j}$ of 1.20 , 1.11 and 1.86 for journey contexts of Business (flows of pedestrians on work-related journeys, excluding those to and from home), Commuting (flows where pedestrians perform the same journey repeatedly, including people moving between home and study/work place) and Leisure (activities such as strolling or shopping).

The overall definition of the step frequency to be used to define pedestrian loads therefore depends upon the attributes of the population using the structure $\left(v_{f}\left(a_{p}, h_{p}\right)\right)$ and the activity and density of the flow $\left(\phi_{j} \phi_{d}\right)$. As an example, distributions of initial step frequencies (ignoring collective behaviour) obtained from this model are shown in Fig. 7 for Western European conditions.

\subsection{Pedestrian weight}

Codes and guidelines currently in use mainly consider flows of pedestrians with a uniform weight of $W_{p}=700 \mathrm{~N}$. However, this pedestrian characteristic differs considerably among subjects according to gender, age and other factors, and therefore this is considered in our model and discussed in Section 6.1. 


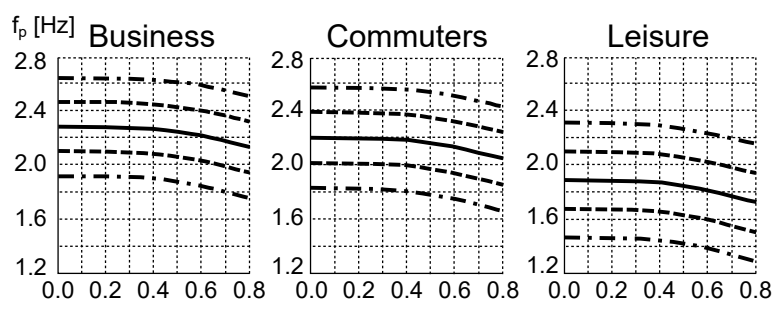

Flow density [pedestrians $/ \mathrm{m}^{2}$ ] $=\mu--\mu_{ \pm} \sigma \quad \cdots \mu_{ \pm 2} \sigma$

Figure 7: Step frequencies $f_{p}$ distribution as a function of flow density.

\subsection{Transverse step width}

The step width, $w_{s, t}$, defined as the total transverse distance between consecutive footsteps $\left(w_{s, t}=2 w_{s}\right)$, is estimated based upon gait characteristics. Data for 294 pedestrians is obtained from research that describes the step width as well as characteristics such age, height, mass and pedestrian velocity. We do not observe any significant dependence of the step width upon age (Ortega et al. 2008), but observe quadratic dependence upon velocity (see Fig. 6c), $v$, and linear dependence upon height, $h_{p}$. The model is again fitted using a weighted regression analysis and is represented by Eq. 8, where the units are $\mathrm{cm}$, $\mathrm{m} / \mathrm{s}$ and $m$ for $w_{s, t}, v$ and $h_{p}$, respectively. The residual standard error of the model is $1.31 \mathrm{~cm}$.

$$
w_{s, t}=26.86-37.74 v+13.37 v^{2}+4.92 h_{p}
$$

\section{Impact of pedestrian intra-subject variability}

Even in the absence of other pedestrians an individual will vary the characteristics of their walking from step-to-step. When observed over a period of time that individual may have well-defined average characteristics, but there will also be an element of inherent variability that is naturally propagated through to the imparted loads.

\subsection{Effect of step frequency variability on vertical response}

From experimental studies, researchers have described the intra-variability of $f_{p}$ as being normallydistributed with standard deviations $\sigma_{f_{p}}$ of $0.037-0.207 \mathrm{~Hz}$ (Maruyama and Nagasaki 1992) and $0.09 \mathrm{~Hz}$ (Butz et al. 2008). While there is conflicting evidence regarding the dependence of $\sigma_{f_{p}}$ upon $v$, the variance of $f_{p}$ is assumed homoscedastic herein. In order to enable step frequencies for individuals to vary for consecutive steps, we make two strong assumptions: a whole sequence of consecutive steps performed by a pedestrian during an event are well-described by a normal distribution; and, sequences of steps are Markovian whereby the properties of each step depend upon the characteristics of the previous step. The first assumption implies a long-term behaviour of the step frequency (being distributed according to $N\left(\mu_{f_{p}}, \sigma_{f_{p}}\right)$, while the second implies a short-term relationship featuring local temporal correlation. The variability of the step frequency is therefore captured using the Metropolis-Hastings algorithm, a Markov chain Monte Carlo (MCMC) method that simulates step lengths according to long and short-term relationships.

Using this MCMC approach, the impact of step frequency variability is investigated for pedestrians using different mean step frequencies. Fig. 8 show the mean and maximum accelerations produced by a single pedestrian walking with a mean step frequency of $\mu_{f_{p}}=1.8 \mathrm{~Hz}$. The results represent the numerical simulation of the passage of this pedestrian across a number of bridges for a range of structureto-pedestrian frequency ratios. The number of steps taken crossing each bridge and the ratio between pedestrian and bridge weight are the same for all analyses. The results shown in the figures are for step

Cite as: Ramos-Moreno, C., A.M. Ruiz-Teran \& P.J. Stafford (2020). Impact of stochastic representations of pedestrian actions on serviceability response. Proceedings of the Institution of Civil Engineers - Bridge Engineering,

https://doi.org/10.1680/jbren.19.00050 

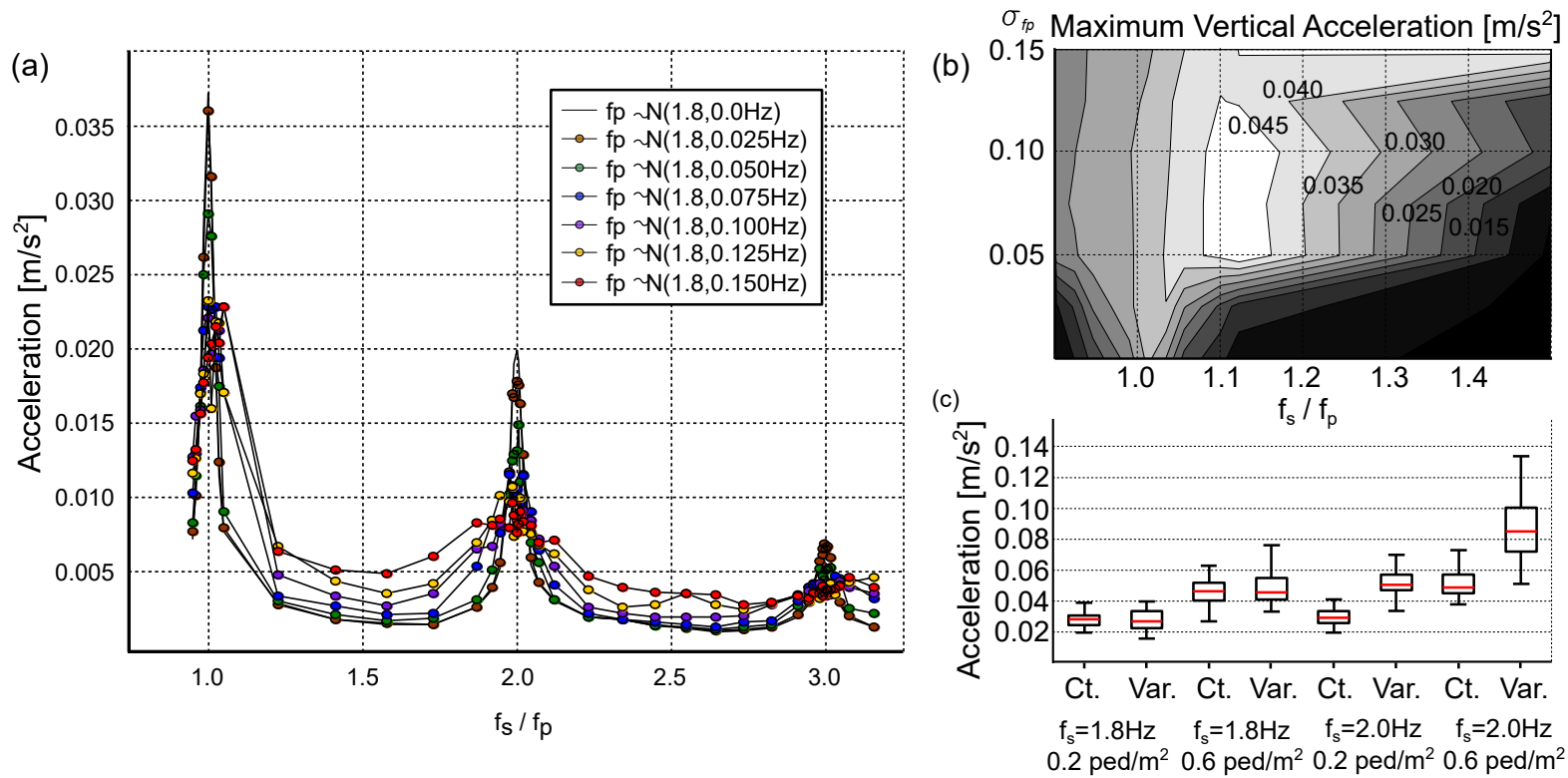

Figure 8: Effects of step frequency variability for individual pedestrians (20 simulations for each scenario): (a) mean vertical accelerations, (b) maximum vertical accelerations around $f_{s}=f_{p}$; and (c) for scenarios with multiple pedestrians (50 simulations for each scenario): maximum vertical accelerations. $C t$. and Var. refer to constant or variable step frequency $f_{p}$.

frequency variabilities ranging from deterministic to the maximum considered realistic, i.e., $\sigma_{f_{p}} \in[0.0,0.15]$ $\mathrm{Hz}$.

For low values of $\sigma_{f_{p}}$ the results are very similar to the deterministic case of pedestrians walking with a constant step frequency (see Fig. 8a). However, for moderate-to-large $\sigma_{f_{p}}$, the peaks associated with resonant conditions are significantly reduced and the troughs between these peaks have significantly increased accelerations. This smoothing of the peaks and troughs arises as the points plotted for a given value of $f_{p}$ really reflect a range of responses at frequencies around this value. In addition, the maximum response no longer occurs for resonant conditions (see Fig. 8b, where accelerations for $f_{s} / f_{p}=1.15$ are more than $30 \%$ larger than accelerations at $f_{s} / f_{p}=1.0$ ). These larger responses for non-resonant conditions are explained by the different impulse transmitted by loads corresponding to different step frequencies (see Fig. $2 b$ and c).

For multiple-pedestrian scenarios, the consideration of the step-frequency variability for individuals strongly affects the response (see Fig. 8c). This figure represents results for crowd flows with densities of 0.2 and $0.6 \mathrm{ped} / \mathrm{m}^{2}$ walking on structures with fundamental vertical frequencies of $f_{s}=1.8 \mathrm{and} 2.0 \mathrm{~Hz}$. To generate the loads for these multi-pedestrian scenarios each pedestrian is first allocated a mean step frequency via sampling from a normal distribution centred on the structural frequency, $\mu_{f_{p}}^{(i)} \sim N\left(f_{s}, 0.175 \mathrm{~Hz}\right)$, with $i$ being an index denoting a particular pedestrian. The intra-subject step frequency variability is then defined using another normal distribution with a mean set to this sampled step frequency. That is, the step frequencies of any individual are represented by $f_{p}^{(i)} \sim N\left(\mu_{f_{p}}^{(i)}, 0.1 \mathrm{~Hz}\right)$, or simply $f_{p}^{(i)}=\mu_{f_{p}}^{(i)}$ in the case that the intra-subject variability is ignored. This standard deviation $\sigma_{f_{p}}=0.10 \mathrm{~Hz}$ is the average of values observed by Butz et al. (2008) and Maruyama and Nagasaki (1992). Step frequency variability produces results that differ from their constant counterparts by an amount that varies with the mean step frequencies of pedestrians. For $\mu_{f_{p}}=1.8 \mathrm{~Hz}$ variable step frequencies give results $5 \%$ larger, while they are $75 \%$ larger for $\mu_{f_{p}}=2.0 \mathrm{~Hz}$. 


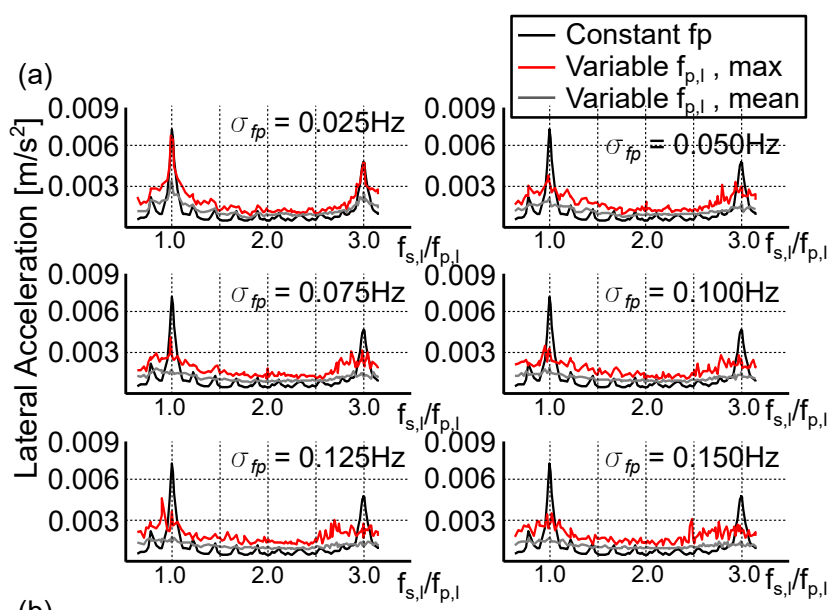

(b)

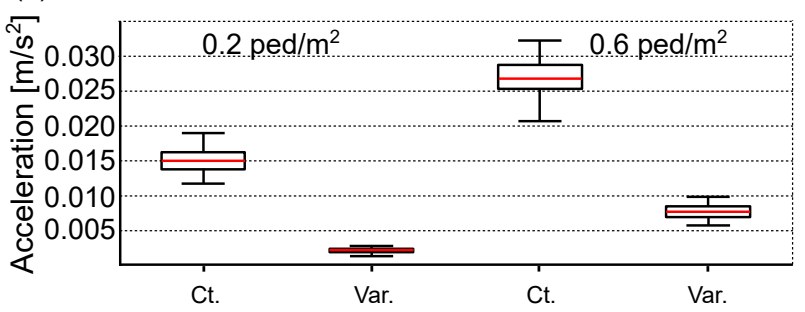

Figure 9: Effects of step frequency variability on lateral response: (a) accelerations caused by a single pedestrian (20 simulations per scenario), (b) maximum lateral accelerations caused by multiple pedestrians scenarios (50 simulations for each scenario). $\sigma_{f_{p}}$ describes the variability of the pedestrian step frequency, and $C t$. and $V a r$. refer to constant or variable lateral step frequency $f_{p, l}$.

\subsection{Effect of step frequency variability on lateral response}

For lateral loads, the effects of the variability of the step frequency of an individual are studied through the same MCMC procedure as implemented for vertical loads, and crossing a range of bridges under the same conditions.

Fig. 9a demonstrates that the effect of the step frequency variability on response is significant, even when relatively small degrees of variability are considered. Generally speaking, both the mean and maximum responses are qualitatively similar and are characterised by their lack of strong peaks that we have previously come to expect when step frequency variability is ignored. This is a very important point as contrary to conventional thinking, the proximity of the lateral step frequency to the lateral structural frequency does not seem to be particularly important once this intra-variability is considered (as long as the lateral movement of the bridge does not trigger a change in the width of the pedestrian step to accommodate for noticeable bridge movement).

When considering results that involve multiple pedestrians, the effects of intra-subject variability are even more dramatic than for single pedestrians (Fig. 9b). For these analyses, the median responses obtained with variable step frequencies are considerably smaller than for the constant frequency case. This variability of step frequency is an important parameter that should be considered when modelling lateral loads.

\subsection{Effect of step width variability on lateral response}

Only a few experimental studies look to characterise the variability of the width of consecutive steps while walking. Helbostad and Moe-Nilssen (2003) suggested that the step width variability has no correlation 


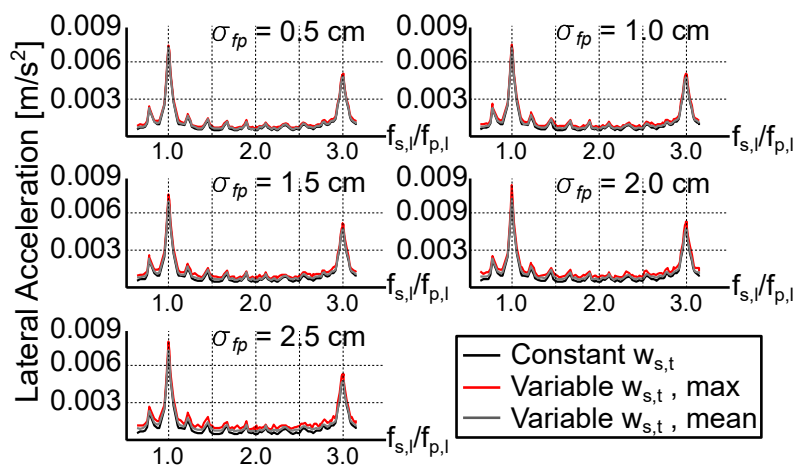

Figure 10: Effects of step width variability on lateral response caused by a single pedestrian (20 simulations per scenario). $\sigma_{f_{p}}$ describes the step width $w_{s, t}$ standard deviation.

with the gait speed and that it increases with the age of the subject. We consider this effect herein using the a MCMC approach, a normal distribution with mean step width of $w_{s, t}=10.3 \mathrm{~cm}$ and a standard deviation ranging from 1.0 to $5.0 \mathrm{~cm}$. In this case, feedback mechanisms, i.e., changes step width resulting from the bridge response, can be neglected due to the small magnitude of the responses generated by the pedestrian lateral loads (see Fig. 10), with maximum lateral accelerations well below $0.01 \mathrm{~m} / \mathrm{s}^{2}$. Fig. 10 suggests that the impact of step width variability is negligible, and we therefore it is ignored from hereafter.

\section{Evaluation of the effects of inter-subject variability}

The purpose of the present section is to focus upon the effect that inter-subject variability of pedestrians in a flow (from subject-to-subject) has upon the acceleration response of a footbridge.

\subsection{Impact of probabilistic description of pedestrian weight}

Codes and guidelines assume a uniform representative weight for all pedestrians. However, a pedestrian's step frequency is related to their weight (through speed, age and height of the subject, as outlined previously) which in turn influences the vertical and horizontal load amplitudes. In order to evaluate the impact of representing pedestrian weight inter-variability we compare results obtained for a constant weight of $700 \mathrm{~N}$ with those when the weight is coupled to pedestrian characteristics such as age, height and speed (using the distribution of weights representative of the UK population, in Digital NHS, where the average weight is $770 \mathrm{~N}$ ). Consistent values of weight, age and height are then used within the equations presented before to define step frequencies, speeds, etc. Values of height and weight are defined according to normal distributions that represent the population depending on age intervals (not including any potential correlations as these are unknown). Values of step frequencies, speeds, etc., have been predicted using the previously proposed expressions without considering any correlation between the parameters (this assumption was formally tested and no significant correlation was found). For a bridge with $f_{s}=2.0 \mathrm{~Hz}$ under a flow of commuting pedestrians with a density $0.6 \mathrm{ped} / \mathrm{m}^{2}$, the accelerations obtained when the inter-subject weight variability is considered are about $10 \%$ greater than for a constant weight, equal to the difference between the mean weight of UK population and the constant weight of codes. The weight variability is therefore unimportant, provided the mean weight used is appropriate. 

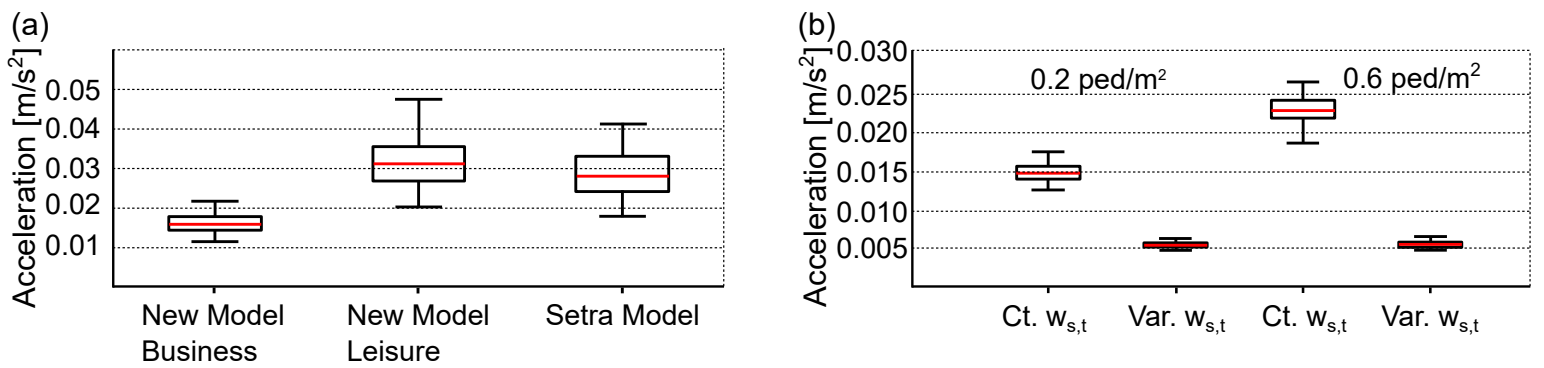

Figure 11: Effects of inter-variability: (a) Maximum vertical midspan accelerations according to traffic type, (b) Accelerations considering step width variability (among pedestrians in a flow). Ct. and Var. refer to constant or variable step width $w_{s, t}$.

\subsection{Impact of probabilistic description of gait characteristics}

The distributions of step frequencies were previously shown to vary with the aim of the journey and flow density (Fig. 7), being substantially different from those considered as critical in guidelines and codes (often using mean values around 1.8-2.0 Hz for all conditions). Fig. 11a provides an example of the differences that can exist under particular circumstances for a structure of $f_{s}=2.0 \mathrm{~Hz}$ under flows of pedestrians with a density of $0.2 \mathrm{ped} / \mathrm{m}^{2}$. The results from the new model are compared with those from the application of the SETRA guideline that assumes that $f_{p} \sim N(2.0,0.175 \mathrm{~Hz})$. The response caused by pedestrians in business conditions are around half of those predicted by the SETRA guideline, whereas those obtained for leisure conditions are $\sim 10 \%$ larger.

These results show how models from guidelines or codes are not able to capture responses caused by pedestrian flows for different contexts. Consequently, it is recommended to consider the proposed distributions in Fig. 7 or to implement models considering journey aims.

\subsection{Impact of probabilistic description of step width}

This section evaluates the relevance of the step width magnitude for multiple pedestrian flows. Two cases are considered: (1) an initial uniform $w_{s, t}=10.3 \mathrm{~cm}$ and (2) an initial non-uniform $w_{s, t}$ (defined by Eq. 8), leading to a normal distribution $\sim N(0.103,0.032 \mathrm{~m})$. In both cases, this step width is modified to satisfy the equilibrium equations of the inverted pendulum. Fig. 11b depicts lateral accelerations of a structure with fundamental lateral frequency $f_{s, l}=1.0 \mathrm{~Hz}$ and pedestrian crowds with densities of 0.2 or $0.6 \mathrm{ped} / \mathrm{m}^{2}$. The variability of step width magnitudes among the different pedestrians in a flow has a very large impact, as the responses for constant step width are three to four times those caused by pedestrians with non-uniform step width. Accordingly, a very detailed evaluation of this magnitude among pedestrians is of utmost importance to predict lateral response of structures under the action of pedestrian flows.

\section{Collective behaviour: pedestrian interactions}

The most critical design scenarios involve multiple pedestrians. Several authors have attempted to account for these loading cases in a simplified manner, through Monte Carlo simulations and relationships between gait characteristics and flow density. However, these simplifications may lead to unrealistic flows for medium and large densities, as the step frequencies adopted by each subject reflect alterations due to changes of speed and trajectory to avoid bumping with other pedestrians. Our model builds upon the work of Helbing et al. (2000), that accounts for such interactions, simulating the two-dimensional movement of subjects over the bridge to obtain the gait parameters of each subject. The parameters required to

Cite as: Ramos-Moreno, C., A.M. Ruiz-Teran \& P.J. Stafford (2020). Impact of stochastic representations of pedestrian actions on serviceability response. Proceedings of the Institution of Civil Engineers - Bridge Engineering.

https://doi.org/10.1680/jbren.19.00050 
define the trajectories of pedestrians are defined in that paper and others such as Willis et al. (2004) or Burgess (1983). The importance of collective behaviour is evaluated by comparing results generated with and without these interactions in a structure with a fundamental vertical frequency of $f_{s}=2,0 \mathrm{~Hz}$ for two different crowd densities (Fig. 12). Three different methodologies are compared: (1) the SETRA guideline, (2) our model considering pedestrian crowds with the same gait characteristics as those defined in the SETRA guideline, and (3) our full model including collective behaviour. As expected, the effects of accounting for crowd interaction are most pronounced for the high pedestrian densities. For the traffic flows with $0.2 \mathrm{ped} / \mathrm{m}^{2}$, the results from the three considered methods have a significant degree of overlap. However, when the density is increased to $0.6 \mathrm{ped} / \mathrm{m}^{2}$ although there remains a reasonable level of consistency in terms of the median predictions, there is a significant difference in the overall range of responses that are observed. Therefore, collective behaviour approaches are important when assessing serviceability limit states.

\section{Summary of proposed model}

The steps to be followed in the proposed model (accounting for intra- and inter-pedestrian variability, crowd and pedestrian-structure interactions) are described herein.

1. According to the design crowd flow density and anticipated mode of traffic for this density, generation of the anthropometric characteristics of the pedestrians in the flow: age, gender (related to the height and mass), height and mass. The mass of each pedestrian could be considered as the mean mass of the described population.

2. From the sampled age, $a_{p}$, and height, $h_{p}$, the average flow density and type of journey, the desired mean walking speed of each pedestrian, $v_{i}$, is computed (considering Eqs. 5-7 and the values of $\phi_{j}$ ) .

3. Using the desired speeds, $v_{i}$, the mean step frequency, $\mu_{f_{p}}^{(i)}$, for each pedestrian, $i$, is obtained using Eq. 4.

4. The initial step width $w_{s, t}$ is then computed using Eq. 8 .

5. The next step is the simulation to account for crowd interactions, the result of which will be trajectories to be followed by each individual subject. The following must be considered:

(a) Pedestrians enter the bridge according to a Poisson arrival process (linked to the flow density) and at a lateral position along the deck width (following an appropriate distribution).

(b) Groups of pedestrians are considered by assigning similar desired speeds, and are forced to remain within a particular fixed distance. The size of the group is generated to reflect the expected characteristics of the flow.

(c) The position and velocity of each pedestrian at any time is obtained using an algorithm such as the social force method described in Helbing et al. (2000). Note that this simulation traces the general path of pedestrians, but not the locations of particular footsteps.

6. Given the first step length, $v_{i} / \mu_{f_{p}}^{(i)}$, the first left or right footfall location is uniformly sampled from the interval $\left[0 ; v_{i} / \mu_{f_{p}}^{(i)}\right]$. The time at which that first step touches the ground is calculated using the pedestrian trajectory.

7. The CoM local trajectory, $y(t)$, for that step is defined solving Eq. 2, at the mid-point of the stance phase (where $y=0$ and $\ddot{y}=0$ ). 


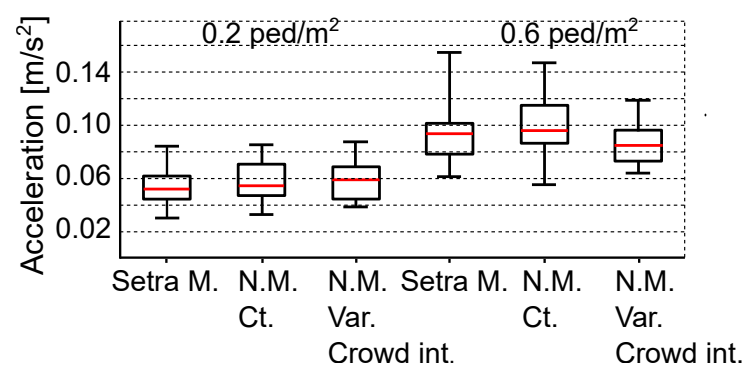

Figure 12: Comparison of results from collective behaviour simulations for two crowd densities ( $C t$. and Var. refer to constant or variable intra-subject step frequency, respectively. Crowd int. refers to collective behaviour, and N.M. refers to the new model).

8. The current step frequency will define the time when the next foot will contact the ground. The step frequency for the following step will be defined by a MCMC sampling to account for intravariability. The mean value for that distribution is given by using Eq. 4 for the speed obtained from the pedestrian trajectory at the time when foot contact is expected.

9. The step width used for the consecutive step and the lateral force to be applied are obtained using the equations of the inverted pendulum model (Eqs. 2-3) and the acceleration felt by this pedestrian through his foot just before the new step. These three final steps will be repeated until the pedestrian crosses the structure.

\section{Conclusions}

The article proposes a realistic, comprehensive, stochastic model for the vertical and lateral loads transmitted by pedestrians while walking over footbridges. The model includes the intra-subject variability associated with human movement, the inter-subject variability among different pedestrians, the variations in flow movement produced when individuals interact in crowds, and pedestrian-structure interaction. Some of these characteristics (not included in load models currently used in practice) have been shown to have a large effect on the response. In this new model the vertical and lateral loads induced by pedestrians are defined in terms of individual footsteps that accurately capture the impulse transmitted by these steps, providing significant benefits over current approaches based upon Fourier series. In addition, new relationships (Eqs. 4, 6 and 8) that accurately link the anthropometric characteristics of each pedestrian to the properties of the load model have been defined, on the basis of a multidisciplinary meta-analysis.

The intra-subject variability of the step frequency has a large impact upon structural response caused by individual pedestrians. Vertical responses for non-resonant conditions may be larger than at resonance, due to the nonlinear scaling of vertical loads with step frequency. The impact of step frequency variability is also considerable for lateral response.

As a result of explicitly modelling the pedestrian and the induced lateral loads influenced by the feedback from the bridge vibration, the response of a structure under the action of a stream of pedestrians is caused by all pedestrians and not only those with step frequencies close to the structural frequency. The lateral loads defined according to this model cannot reproduce a change of velocity and step frequency due to the movement of the footbridge noticed by the pedestrians.

Inter-subject variability is also important. The predicted accelerations are sensitive to the gait characteristics (speed, step frequency and step width) attributed to each pedestrian in a flow. These should be define according to the situation considered (type of flow and densities most likely to occur).

Cite as: Ramos-Moreno, C., A.M. Ruiz-Teran \& P.J. Stafford (2020). Impact of stochastic representations of pedestrian actions on serviceability response. Proceedings of the Institution of Civil Engineers - Bridge Engineering.

https://doi.org/10.1680/jbren.19.00050 
For medium and large pedestrian densities, there is a significant interaction between the pedestrians affecting their trajectories, speeds and frequencies and, in turn, the bridge response. Therefore, collective behaviour should be realistically and accurately modelled using sophisticated crowd simulations.

This sophisticated load model has been used to reproduce accelerations experimentally recorded in real bridges (e.g., a multispan postensioned concrete footbridge located in Iceland, Gudmundsson et al. 2008, or a three span steel box girder in Portugal, Alves et al. 2008), showing that it can predict results with a large degree of accuracy (differences below $5 \%$ in both cases).

The proposed model entails the development of sophisticated and substantial work which may be required when there is a critical need to accurately assess the serviceability performance of a structure during detailed design, prior to the construction stage. The model proposed in this paper is complex for direct implementation with computer analyses and, in fact, not many software packages for structural analysis currently used by designers would allow its implementation. Nonetheless, the paper describes a method that can be implemented by developers of software packages as subroutines that are user friendly and include all the analyses in the background.

\section{Acknowledgments}

The authors are grateful for the support received by the first author from "La Caixa" Foundation to fund her PhD studies at Imperial College London.

\section{References}

Alves R, Barbosa F \& Caetano E (2008), The Aldeias Footbridge at Gouveia: design, construction and dynamic behaviour, in E. Caetano \& A. Cunha, eds, Proceedings of the Third International Conference, Footbridge 2008.

Bachmann H (1992), Case studies of structures with man-induced vibrations, Journal of Structural Engineering 118(3): $631-647$.

Bachmann H \& Ammann WJ (1987), Vibration in structures: Induced by man and machines, Structural Engineering Documents 3e, IABSE, Zurich.

Bachmann H, Pretlove AJ \& Rainer JH (1995), Vibrations induced by people, Vibration Problems in Structures. Practical Guidelines, Birkhäuser Verlag, Basel, Switzerland.

Bertram JEA \& Ruina A (2001), Multiple walking speed-frequency relations are predicted by constrained optimization, Journal of Theoretical Biology 209: 445-453.

Blanchard J, Davies BL \& Smith JW (1977), Design criteria and analysis for dynamic loading of footbridges, in Proceedings of a Symposium on Dynamic Behaviour of Bridges at the Transport and Road Research Laboratory, TRRL, Crowthorne, Berkshire, UK, pp. 90-106.

Brownjohn JMW, Pavic A \& Omenzetter P (2004), A spectral density approach for modelling continuous vertical forces on pedestrian structures due to walking, Canadian Journal of Civil Engineering 31(1): 65-77.

BSI (British Standards Institution) (1978), BS 5400: Part 2. Appendix C: Vibration serviceability requirements for foot and cycle track bridges, BSI, London, UK.

BSI (British Standards Institution) (2006), BS 5400:Part 2. Annex B: Vibration serviceability requirements for foot and cycle track bridges, BSI, London, UK.

BSI (British Standards Institution) (2008), NA to BS EN 1991-2:2003. UK National Annex to Eurocode 1: Actions on structures. Part 2: Traffic loads on bridges, BSI, London, UK.

Burgess JW (1983), Interpersonal spacing between surrounding nearest neighbors reflects both familiarity and environmental density, Ethology and Sociobiology 4: 11-17.

Cite as: Ramos-Moreno, C., A.M. Ruiz-Teran \& P.J. Stafford (2020). Impact of stochastic representations of pedestrian actions on serviceability response. Proceedings of the Institution of Civil Engineers - Bridge Engineering,

https://doi.org/10.1680/jbren.19.00050 
Butz C, Feldmann M, Heinemeyer C \& et al. (2008), Advanced load models for synchronous pedestrian excitation and optimised design guidelines for steel footbridges, Research Fund for Coal and Steel.

Carroll SP, Owen JS \& Hussein MFM (2013), A coupled biomechanical/discrete element crowd model of crowdbridge dynamic interaction and application to the Clifton suspension bridge, Engineering Structures 49: 58-75.

Charles P, Hoorpah W, Boniface V \& et al. (2006), Footbridges. Assessment of vibrational behaviour of footbridges under pedestrian loading. Practical guidelines, SETRA/AFGC.

Chen J, Ding G \& Živanović S (2019), Stochastic single footfall trace model for pedestrian walking load, International Journal of Structural Stability and Dynamics 19(3).

Dallard P, Fitzpatrick AJ, Flint A, Le Bourva S, Low A, Ridsdill Smith RM \& Willford M (2001), The London Millennium Footbridge, The Structural Engineer 79: 17-33.

Digital NHS (accessed 2013), Health Survey for England - 2011 Digital NHS, UK.

URL: http://digital.nhs.uk

Eames MHA, Cosgrove A \& Baker R (1999), Comparing methods of estimating the total body centre of mass in three-dimensions in normal and pathological gaits, Human Movement Science 18: 637-646.

Ebrahimpour A \& Sack RL (1992), Design live loads for coherent crowd harmonic movements, Journal of Structural Engineering 118(4): 1121-1136.

Ellingwood B \& Tallin A (1984), Structural Serviceability: Floor Vibrations, Journal of Structural Engineering 110(2): 401-418.

Fujino P, Pacheco BM, Nakamura S \& Warnitchai S (1993), Synchronization of human walking observed during lateral vibration of a congested pedestrian bridge, Earthquake Engineering \& Structural Dynamics 22(9): 741758.

Gard SA \& Childress DS (2001), What determines the vertical displacement of the body during normal walking?, JPO Journal of Prosthetics \& Orthotics 13(3): 64-67.

Giakas G \& Baltzopoulos V (1977), Time and frequency domain analysis of ground reaction forces during walking: an investigation of variability and symmetry, Gait and Posture 5(3): 189-197.

Gudmundsson G, Ingólfsson ET, Einarsson B \& Bessason B (2008), Serviceability assessment of three lively footbridges in Reykjavik, in E. Caetano \& A. Cunha, eds, Proceedings of the Third International Conference, Footbridge 2008, Porto, Portugal, 2008.

Helbing D, Farkas I \& Vicsek T (2000), Simulating dynamical features of escape panic, Nature 407: 487-490.

Helbostad JL \& Moe-Nilssen R (2003), The effect of gait speed on lateral balance control during walking in healthy elderly, Gait and Posture 18: 27-36.

Ingólfsson ET \& Georgakis CT (2011), A stochastic load model for pedestrian-induced lateral forces on footbridges, Engineering Structures 33(12): 3454-3470.

Ingólfsson ET, Georgakis CT, Ricciardelli F \& Jonsson J (2011), Experimental identification of pedestrian-induced lateral forces on footbridges, Journal of Sound and Vibration 330: 1265-1284.

Ingólfsson ET, Gudmundsson GV, Živanović S \& Pavic A (2012), Crowd-induced vibrations of a steel footbridge in Reykjavik, in IMAC XXX: Conference and Exhibition on Structural Dynamics, Jacksonville, Florida, USA, Vol. 26, pp. 61-72.

Keller TS, Weisberger AM, Ray J L, Hasan SS, Shiavi RG \& Spengler DM (1996), Relationship between vertical ground reaction force and speed during walking, slow jogging, and running, Clinical Biomechanics 11: 253-259.

Kerr SC (1998), Human Induced Loading on Staircases, PhD thesis, University of London, London, UK.

Cite as: Ramos-Moreno, C., A.M. Ruiz-Teran \& P.J. Stafford (2020). Impact of stochastic representations of pedestrian actions on serviceability response. Proceedings of the Institution of Civil Engineers - Bridge Engineering,

https://doi.org/10.1680/jbren.19.00050 
Macdonald JHG (2009), Lateral excitation of bridges by balancing pedestrians, Proceedings of the Royal Society A-Mathematical Physical and Engineering Sciences 465: 1055-1073.

Mackinnon CD \& Winter D A (1993), Control of whole body balance in the frontal plane during human walking, Journal of Biomechanics 26: 633-644.

Maruyama H \& Nagasaki H (1992), Temporal variability in the phase durations during treadmill walking, Human Movement Science 11: 335-348.

Matsumoto Y, Nishioka T, Shiojiri H \& Matsuzaki K (1978), Dynamic Design of Footbridges, IABSE Proceedings P-17/78: 21-52.

Millford M R \& Young P (2006), A Design Guide for Footfall Induced Vibration of Structures, The Concrete Centre.

Mulas MG, Lai E \& Lastrico G (2018), Coupled analysis of footbridge-pedestrian dynamic interaction, Engineering Structures 176(1): 127-142.

Nilsson J \& Thorstensson A (1987), Adaptability in frequency and amplitude of leg movements during human locomotion at different speeds, Acta Physiologica Scandinavica 129: 107-114.

Nilsson J \& Thorstensson A (1989), Ground reaction forces at different speeds of human walking and running, Acta Physiologica Scandinavica 136(2): 217-227.

Ortega JD, Fehlman LA \& Farley CT (2008), Effects of aging and arm swing on the metabolic cost of stability in human walking, Journal of Biomechanics 41: 3303-3308.

Pedersen L \& Frier C (2010), Sensitivity of footbridge vibrations to stochastic walking parameters, Journal of Sound and Vibration 329(13): 2683-2701.

Ped-net.org (accessed 2013).

URL: https://www.ped-net.org

Rainer JH \& Pernica G (1986), Vertical dynamic forces from footsteps, Canadian Acoustics 14(2): 12-21.

Ramos-Moreno C (2016), Design of cable-stayed footbridges under serviceability loads, PhD thesis, Imperial College London, Department of Civil and Environmental Engineering, London, UK.

Ricciardelli F \& Pizzimenti AD (2007), Lateral walking-induced forces on footbridges, Journal of Bridge Engineering 12(6): $677-688$.

Rose, J. \& Gamble, J., eds (1994), Human walking, Williams \& Wilkins, Philadelphia, USA.

Townsend M (1985), Biped gait stabilization via foot placement, Journal of Biomechanics 18: 21-38.

Venuti F, Bruno L \& Bellomo N (2007), Crowd dynamics on a moving platform: Mathematical modelling and application to lively footbridges, Mathematical and Computer Modelling 45(3-4): 252-269.

Venuti F, Bruno L \& Napoli P (2007), Pedestrian lateral action on lively footbridges: A new load model, Structural Engineering International 17(3): 236-241.

Weidmann U (1993), Transporttechnik der Fussgänger - Transporttechnische Eigenschaften des Fussgängerverkhers (Literaturauswertung). [In German], Institut für Verkehrsplanung, Transporttechnick, Strassen und Eisenbahnbau, ETH Zurich.

Willis A, Gjersoe N, Havard C, Kerridge J \& Kukla R (2004), Human movement behaviour in urban spaces: implications for the design and modelling of effective pedestrian enviroments, Environment and Planning B: Planning and Design 31.6: 805-828.

Zivanović S, Pavic A \& Reynolds P (2007), Probability-based prediction of multi-mode vibration response to walking excitation, Engineering Structures 29(6): 942-954.

Cite as: Ramos-Moreno, C., A.M. Ruiz-Teran \& P.J. Stafford (2020). Impact of stochastic representations of pedestrian actions on serviceability response. Proceedings of the Institution of Civil Engineers - Bridge Engineering,

https://doi.org/10.1680/jbren.19.00050 